\title{
Enhanced sunflower optimization for placement distributed generation in distribution system
}

\author{
Thuan Thanh Nguyen \\ Faculty of Electrical Engineering Technology, Industrial University of Ho Chi Minh City, Viet Nam
}

\begin{tabular}{|c|c|}
\hline Article Info & ABSTRACT \\
\hline Article history: & \multirow{9}{*}{$\begin{array}{l}\text { Installation of distribution generation (DG) in the distribution system gains } \\
\text { many technical benefits. To obtain more benefits, the location and size of DG } \\
\text { must be selected with the appropriate values. This paper presents a method } \\
\text { for optimizing location and size of DG in the distribution system based } \\
\text { on enhanced sunflower optimization (ESFO) to minimize power loss of } \\
\text { the system. In which, based on the operational mechanisms of the original } \\
\text { sunflower optimization (SFO), a mutation technique is added for updating } \\
\text { the best plant. The calculated results on the } 33 \text { nodes test system have shown } \\
\text { that ESFO has proficiency for determining the best location and size of DG } \\
\text { with higher quality than SFO. The compared results with the previous } \\
\text { methods have also shown that ESFO outperforms to other methods in term of } \\
\text { power loss reduction. As a result, ESFO is a reliable approach for the DG } \\
\text { optimization problem. }\end{array}$} \\
\hline Received Apr 25, 2020 & \\
\hline Revised Jun 20, 2020 & \\
\hline Accepted Jul 1, 2020 & \\
\hline Keywords: & \\
\hline & \\
\hline Enhanced sunflower & \\
\hline optimization & \\
\hline Location and size & \\
\hline Power loss & \\
\hline
\end{tabular}

Power loss

Sunflower optimization

\section{Corresponding Author:}

Thuan Thanh Nguyen,

Faculty of Electrical Engineering Technology,

Industrial University of Ho Chi Minh City,

No. 12 Nguyen Van Bao, Ward 4, Go Vap District, Ho Chi Minh City, Viet Nam.

Email: nguyenthanhthuan@iuh.edu.vn
This is an open access article under the CC BY-SA license.

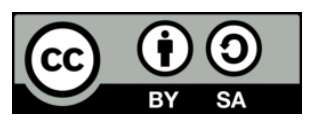

\section{INTRODUCTION}

Distributed generations (DG) is small power plant connected to the power system at distribution voltage level or installed closed to customers [1]. From the operational perspective, DG installation is able to bring many technical benefits for distribution network such as power loss reduction, voltage improvement and reliability enhancing. However, these maximum benefits are only achieved when DG is installed in the proper position as well as the appropriate capacity, otherwise wrong position and size of DG may cause more technical issues. Therefore, optimization of location and size of DG is the problem that is attracted by many concerns.

For solving the DG optimization problem, there are various methods that have been proposed. In [2], genetic algorithm (GA) is proposed to find the optimal location and size of DG to gain more revenues and reduce imposed costs [2]. In [3], GA is used for solving the DG optimization problem to reduce power loss. Similarly, in [4], GA is also proposed for determining location and size of DG in the smart grid network. In [5], artificial bee colony method (ABC) has been applied to find the appropriate position and size of DG in the distribution system. In [6], power loss reduction is minimized by installing DG based on honey bee mating optimization (HBMO). In [7], particle swarm optimization (PSO) is combined with GA for optimization of DG to reduce power loss and enahnce voltage stability. In [8], PSO is proposed to solve the the DG optimization problem combined with the network reconfiguration.

To solve the DG optimization problem, not only common methods such as GA, ABC, HBMO and PSO are used, but also many recently developed algorithms have been successfully applied such as whale 
optimization algorithm (WOA) [9, 10], harmony search (HS) [11, 12], modified crow search (MCS) [13], adaptive cuckoo search (ACS) [14], fireworks algorithm (FA) [15], coyote algorithm [16], uniform voltage distribution algorithm (UVD) [17], hyper cube ant colony optimization (HCACO) [18], runner root [19] and modified plant growth simulation (MPGS) [20]. Compared with classical methods such as dynamic programming [21], linear programming [22] and mixed integer linear programming [23], methods based on general knowledge such as GA, ABC and the aforementioned methods often get better quality results when applied to the DG optimization problem. Therefore, researching new methods to apply to the DG optimization problem is also a matter of concern to supplement the potential methods for solving the problem.

This paper presents a method to optimize location and size of DG in the distribution system to minimize power loss based on enhanced sunflower optimization (ESFO). Wherein, ESFO is enhanced from the original sunflower optimization (SFO) [24]. In [24], the original SFO is taken from an ideal of movement of the sunflower plant to take sunlight. In order to apply for solving the optimization problem, each sunflower plant is considered as solution. The best plant is examined as the sun and all of other plants will move to the best one. Based on the mechanisms of creating new plants of SFO, we proposed to add the mutation technique to create a new plant by mutating the best plant for updating the best sunflower plant.

The effectiveness of the proposed ESFO has been demonstrated on the 33 nodes test distribution system. The calculated result is compared to that of SFO and other previous methods. The contributions of this work can be highlighted as follows:

- A mutation of creating a new plant for updating the best sunflower plant is proposed for ESFO.

- ESFO is first proposed for the DG optimization problem.

- The effectiveness of ESFO is evaluated on the 33 nodes test distribution system.

- ESFO is more effective than SFO as well as the previous methods for the DG optimization problem in terms of the obtained solution quality.

The rest paper is organized as follows: The following section shows the problem of DG optimization. The section 3 demonstrates the proposed ESFO and its application for the DG optimization problem. The section 4 presents results and discussion. Finaly, the conclusion section is demonstrated.

\section{PROBLEM OF DG OPTIMIZATION}

One of the biggest benefits of installing DG in the distribution system is power loss reduction. The main goal of the problem is to minimize power loss. It is determined as follows:

$$
\Delta P=\sum_{i=1}^{n_{l}} p_{\text {loss }, i}
$$

where $p_{\text {loss }, i}$ is power loss of the line $i . n_{l}$ is number of lines in the system.

Installing DG in the distribution system should be maintained the following constraints:

- Voltage and current limits:

$$
\left\{\begin{array}{l}
\mathrm{V}_{\min }^{l i} \leq V_{i} \leq \mathrm{V}_{\text {max }}^{l i} \\
L I_{i} \leq L I_{\max , i}^{l i} \quad ; i=1 \div n_{b}
\end{array}\right.
$$

where $V_{\min }^{l i}$ and $V_{\text {max }}^{l i}$ are the lower and upper limits of the node voltage. $V_{i}$ is the voltage amplitude of node $i$. $L I_{i}$ and $L I_{\max , i}^{l i}$ are the load carrying coefficient and maximum coefficient of the line $i$. $n_{b}$ is number of nodes in the system.

- DG size limits:

$$
s_{k} \leq s_{k, \max } ; k=1 \div n_{d g}
$$

where $s_{k}$ is size of DG $k$ in MW. $s_{k, \max }$ is the maximum capacity limit of DG $k . n_{d g}$ is number of DG installed in the distribution system.

\section{ENHANCED SUNFLOWER OPTIMIZATION FOR THE DG OPTIMIZATION PROBLEM}

\subsection{The original sunflower optimization}

For solving the optimal problem, the population of sunflower plants is updated by three different techniques. The first one is called pollination technique. A part of the population that is determined by the pollination rate is chosen for pollination and creating new plants for the next generation. The pollination technique to create new plants is done by the combination of two consecutive plants in the population as follows:

Int J Elec \& Comp Eng, Vol. 11, No. 1, February 2021: 107 - 113 


$$
p_{i}=\operatorname{rand}(0,1) \cdot\left(p_{i}-p_{i+1}\right)+p_{i+1} ; i=1 \div r_{p} \cdot n
$$

where $p_{i}$ and $p_{i+1}$ are the plant $i$ and $i+1$ in the population. $r_{p}$ is the pollination rate that is chosen to 0.6 [24]. $n$ is number of plants in the population.

The second one is called the survival technique. In the remainder of the population, a number of plants will survive and maintain over the next generation. The retention of information of a plant through the next generation is determined by the distance from itself to the best plant. The closer to the best one a plant is, the greater probability that it will remain the same over the next generation. The details of creating new plants by the survival technique is described as below:

$$
p_{i}=p_{i}+\operatorname{rand}(0,1) \cdot\left(\left(p_{\text {best }}-p_{i}\right) /\left(\left\|p_{\text {best }}-p_{i}\right\|\right)\right) ; i=r_{p} \cdot n \div n \cdot\left(1-r_{d}\right)
$$

where, $p_{\text {best }}$ is the best plant. $\left\|p_{\text {best }}-p_{i}\right\|$ is the Euclidean length of the $p_{\text {best }}-p_{i}$ vector. $r_{d}$ is the death rate that is chosen to 0.1 [24].

The last one is called mortality technique. In the remainder of the population, a number of plants that is determined by the mortality rate will be died and replaced by new random plants as follows:

$$
p_{i}=u b+\operatorname{rand}(0,1) \cdot(u b-l b) ; i=n \cdot\left(1-r_{d}\right) \div n
$$

where $u b$ and $l b$ are the upper and lower bounds of the plants.

The new plants are validated the fitness function and they are used to replace for the corresponding ones in the population if their quality is better than the corresponding ones' quality. In addition, the best sunflower plant is updated until the maximum number of generation is reached.

\subsection{The enhanced sunflower optimization}

It can be seen that SFO uses three different techniques to renew the population. In the first technique, the method of combining two consecutive plants in the population to create a new plant. The role of this technique is exploration and exploitation the search space. The second technique helps to create new plants that move to the best plant. It helps SFO to exploit the search space. Meanwhile, the final technique generates random plants to explore the search space and prevent the SFO from converging to local optimal solution soon. Based on the above mechanisms of SFO, in this study we propose the enhanced sunflower optimization (ESFO). Wherein, a new technique is suggested to create a new plant by mutating the best one. After the new population of plants has been created from the three above techniques, their fitness function is calculated and the best plant is determined. Before the population is renewed again in the next generation, the new plant is created by mutation of the best plant. If the new plant has better quality than the best one, it is used to replace the best plant, otherwise it will be die if its quality is worse than the best one. The new plant is generated as follows:

$$
p_{\text {new }, j}=p_{\text {best }, j}+\operatorname{rand}(0,1) \cdot \mu \cdot \rho(0,1) ; j=1 \div d
$$

where, $p_{n e w, j}$ and $p_{\text {best }, j}$ are the control variable $j$ of the new and best plants. $d$ is problem dimension. $\mu$ is a constant to determine the maximum change limit of the variable. $\rho(0,1)$ is a function that returns the value of 0 or 1 . If the $\rho(0,1)$ is equal to 0 , the $p_{n e w, j}$ is similar to $p_{b e s t, j}$ otherwise the $p_{n e w, j}$ will be set to new value. The value of $\rho(0,1)$ is determined as follows:

$$
\rho(0,1)=\left\{\begin{array}{l}
1 ; \text { if rand }(0,1)<r_{m} \\
0 ; \text { otherwise }
\end{array}\right.
$$

where $r_{m}$ is mutation rate that is selected to 0.2 . It means that about twenty percent of variables of the $p_{\text {best }}$ is renewed. The new plant is validated the fitness function and it will become to the best plant if it has better quality than the best one, it is used to replace the best plant, otherwise it will be die.

\subsection{The application of ESFO for the DG optimization problem}

Step 1: Initialization

For applying to the DG optimization problem, each sunflower plant is presented as follows:

$$
p_{i}=\left[l_{i, k}, s_{i, k}\right] ; i=1 \div n \text { and } k=1 \div n_{d g}
$$


where $l_{k}$ and $s_{k}$ are location and size of DG $k . n_{d g}$ is number of DG installed in the distribution system.

At the beginning, the initial plants are created randomly as follows:

$$
p_{i}=u b+\operatorname{rand}(0,1) \cdot(u b-l b) ; i=1 \div n
$$

where $u b$ and $l b$ for are determined as follows:

$$
\left\{\begin{array}{l}
u b=\left[l_{k, \text { max }}, s_{k, \text { max }}\right] \\
l b=\left[l_{k, \text { min }}, s_{k, \text { min }}\right]
\end{array} ; k=1 \div n_{d g}\right.
$$

where $s_{k, \max }$ and $s_{k, \min }$ are the upper and lower sizes of the DG $k . l_{k, \max }$ and $l_{k, \min }$ are the highest and lowest nodes in the distribution system.

Then, the variable part indicates the location of DG is rounded to integer value to map with the DG optimization problem and the system data is updated to calculate the fitness function $(F)$ value as follows:

$$
F i t=\Delta P+K \cdot\left[\begin{array}{c}
\max \left(\mathrm{V}_{\min }^{l i}-V_{\min }, 0\right)+\max \left(V_{\max }-\mathrm{V}_{\text {max }}^{l i}, 0\right) \\
+\max \left(L I_{\max }-\mathrm{LI}_{\text {max }}^{l i}, 0\right)
\end{array}\right]
$$

where, $\mathrm{K}$ is penalty coefficient, $V_{\min }$ and $V_{\max }$ are the minimum and maximum voltage amplitude is the system. $L I_{\max }$ maximum load carrying coefficient in the system.

Based on the $F$ value, the best plant $p_{\text {best }}$ is determined.

Step 2: Creating of new plants by using the pollination, survival and mortality techniques

The new population of sunflower plants is generated by using the pollination, survival and mortality techniques as described in equations (4-6). Wherein, the variable part indicates the location of DG of new plants is rounded to integer value. The quality of new plants is validated by the fitness function as (12). The new plants are used to replace for the corresponding ones in the current population if their quality is better than the corresponding ones' quality. Otherwise, they will be died. The final procedure of this step is to update the best plant by comparing the plant having the best fitness value with the best plant of the previous generation.

Step 3: Creating the new plant by mutating the best plant

From the best plant determined in step 2, the new plant is created by using equation (7). Then, the variable part indicates the location of DG of new plants is rounded to integer value to map with the DG optimization problem. Finally, its quality evaluated by using (12) is compared with the best plant. The best plant is updated one more time if the quality of the new plant is better than that of the best plant.

Step 4: Checking the stopping condition

The stopping condition is set based on a maximum number of fitness evaluation (MNFE). It means that step 2 and 3 will be executed until number of fitness evaluation reaches to the MNFE value.

\section{RESULTS AND DISCUSSION}

In this section, the performance of ESFO is compared with the original SFO in the same personal computer based on Matlab platform. In addition, the effectiveness of ESFO is also compared with previous DG optimization methods in the literature. All of methods are validated on the 33 nodes distribution system as shown in Figure 1 [25]. In which, the load, voltage level and line parameters are taken from [25] and the rated current of all lines are referenced set to $150 \mathrm{~A}$. The number of DG selected for optimization is fixed to 3 and their power limit is set to $2 \mathrm{MW}$. The parameters of ESFO and SFO consisting of $n$ and MNFE are set to 30 and 9000, respectively. For ESFO, the $\mu$ constant in (7) is chosen to 3 . The penalty coefficient $\mathrm{K}$ in the fitness function equation is selected to 1000 .

The calculated results of ESFO are shown in Table 1. From the table, the total losses have been reduced from $202.6863 \mathrm{~kW}$ to $72.2671 \mathrm{~kW}$. It means that the power loss reduction is 130.4192 corresponding to reduction of $64.35 \%$. Furthermore, the node voltage and line current as shown in Figure 2 have been also enhanced after installing three DG with power of 1.0945, 1.3162 and $0.8046 \mathrm{MW}$ at the nodes of 30, 24 and 13. In more details, the minimum voltage amplitude in per unit has been raised from 0.9131 to 0.9709 and the maximum LI value has been decreased from 1.4024 to 0.7354 . In comparison with SFO, power loss obtained by ESFO is $0.4715 \mathrm{~kW}$ lower than that of SFO corresponding to $0.24 \%$ reduction higher than that of SFO. In addition, the minimum voltage amplitude obtained by ESFO is 0.003 higher and the maximum LI value is 0.064 lower than those of SFO. The improvement of voltage profile of SFO shown in Figure 2 is also 
worse than that of ESFO. These results show that ESFO outperforms to SFO for finding optimal location and size of DG in the distribution system. The optimal location of three DG in the system is shown in Figure 3.

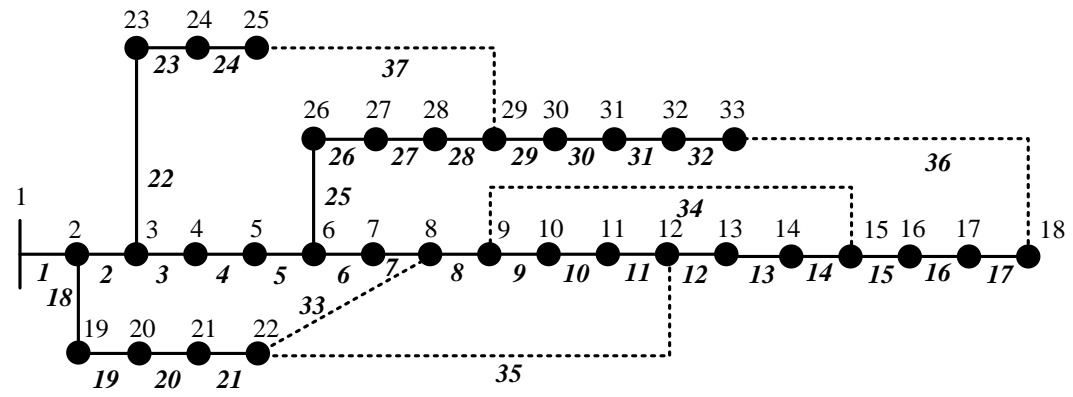

Figure 1. The 33 nodes test system

Table 1. Optimal solution for the 33 nodes system

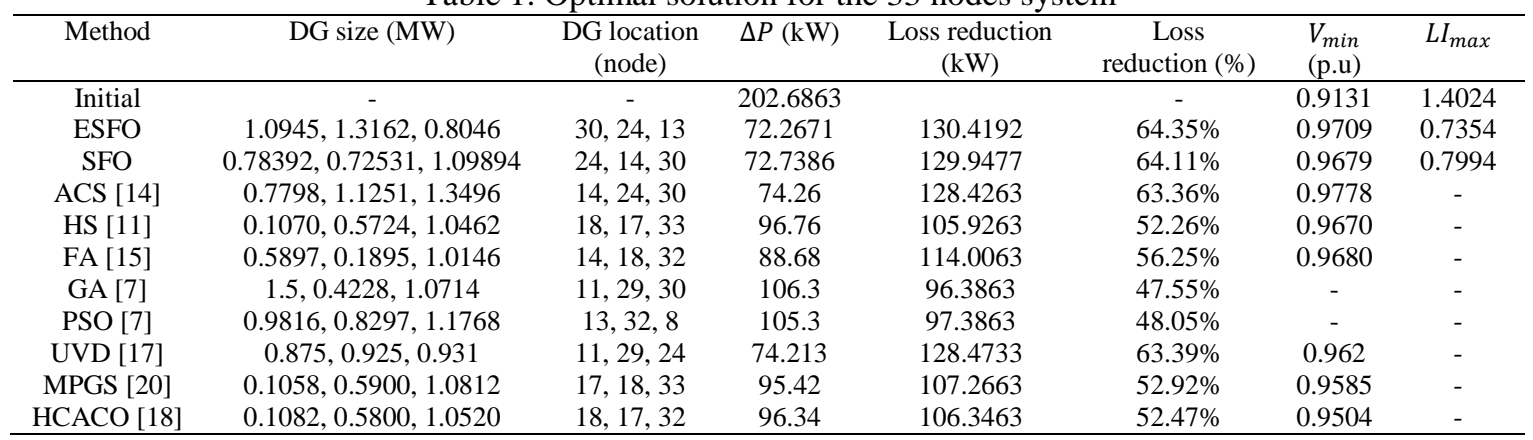

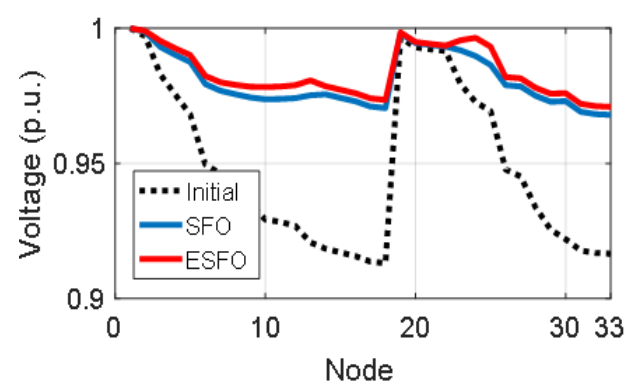

(a)

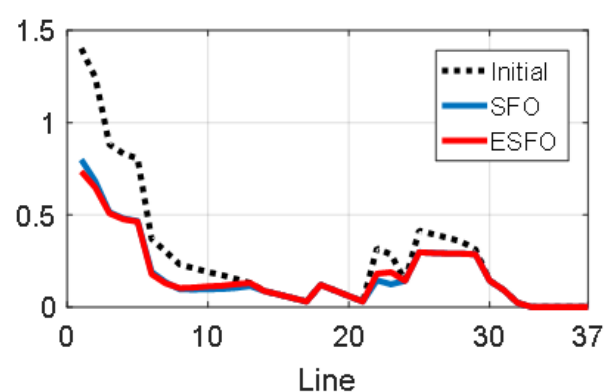

(b)

Figure 2. The voltage and current profile after optimizing DG by ESFO and SFO, (a) Voltage profile and (b) Current profile

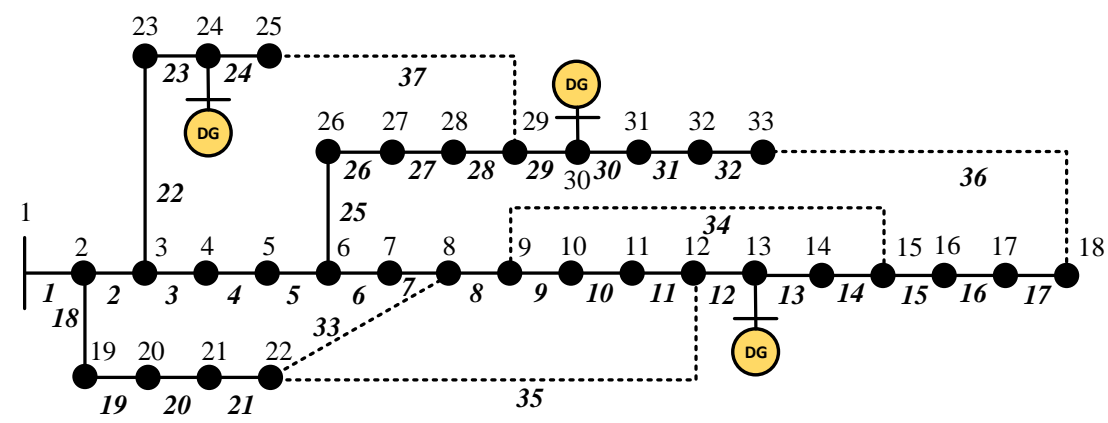

Figure 3. Location of DG obtained by ESFO 
Compared to results obtained by the previous methods ACS [14], HS [11], FA [15], GA [7], PSO [7], UVD [17], MPGS [20] and HCACO [18], power loss reduction obtained by the proposed method is $0.99 \%, 12.09 \%, 8.10 \%, 16.80 \%, 16.30 \%, 0.96 \%, 11.43 \%$ and $11.88 \%$ higher than that of the above methods. Table 2 also shows that the minimum voltage amplitude obtained by ESFO is better than that of most of methods except the ACS [14].

Table 2. Comapred results between ESFO and SFO for the DG optimization problem

\begin{tabular}{|c|c|c|c|c|c|c|}
\hline Method & Fit $\max$ & Fit $_{\text {min }}$ & Fit $_{\text {mean }}$ & STD & $G_{\text {mean }}$ & Run times (s) \\
\hline ESFO & 78.6518 & 72.2671 & 74.8274 & 1.6571 & 172 & 30.2619 \\
\hline SFO & 81.1677 & 72.7386 & 76.1131 & 2.4050 & 173 & 29.1834 \\
\hline
\end{tabular}

In order to compare the performance of ESFO and SFO, both of the algorithms are executed in 50 independent runs and the obtained indexes comprising of maximum $\left(\right.$ Fit $\left._{\text {max }}\right)$, minimum $\left(F_{i t_{\text {min }}}\right)$, mean $\left(\right.$ Fit $\left._{\text {mean }}\right)$ and standard deviation $(S T D)$ as well as the mean of number of convergence generations $\left(G_{\text {mean }}\right)$ are used to evaluate the effectiveness of two methods. The obtained results are presented in Table 2. From the table, the $F i t_{\text {max }}, F i t_{\text {min }}$, Fit $t_{\text {mean }}$ and STD values obtained by ESFO are better than those of SFO. Wherein, these values of ESFO are 2.5159, 0.4715, 1.2857 and 0.7479 lower than those of SFO, respectively. Meanwhile, the run time value of ESFO is only 1.0785 longer than that of SFO. In addition, the minimum fitness value obtained in each run as shown in Figure $4 \mathrm{a}$ shows that the better performance of ESFO compared to SFO. In which, there are 35 runs in total 50 runs, ESFO obtained a better fitness value than that of SFO while SFO has only found a better fitness value than that of ESFO in 15 runs. The maximum, minimum and mean convergence curves of ESFO and SFO in 50 runs are shown in Figure 4b. From all curves obtained by ESFO are much lower than corresponding ones of SFO. This again confirms that ESFO's improvements have yielded more positive results than SFO for the DG optimization problem.

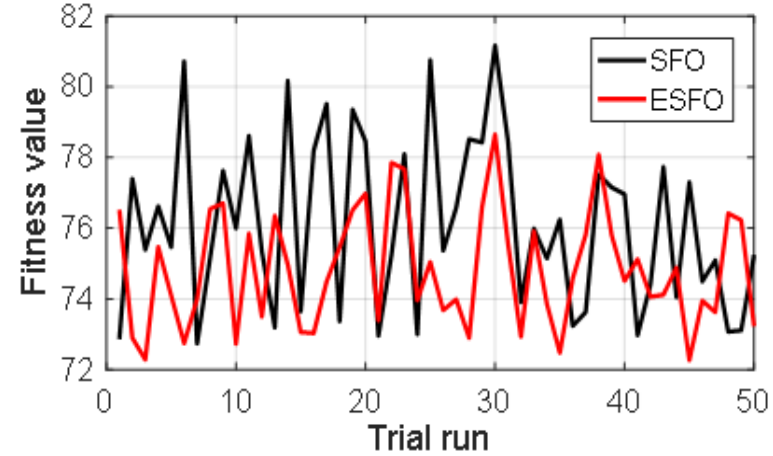

(a)

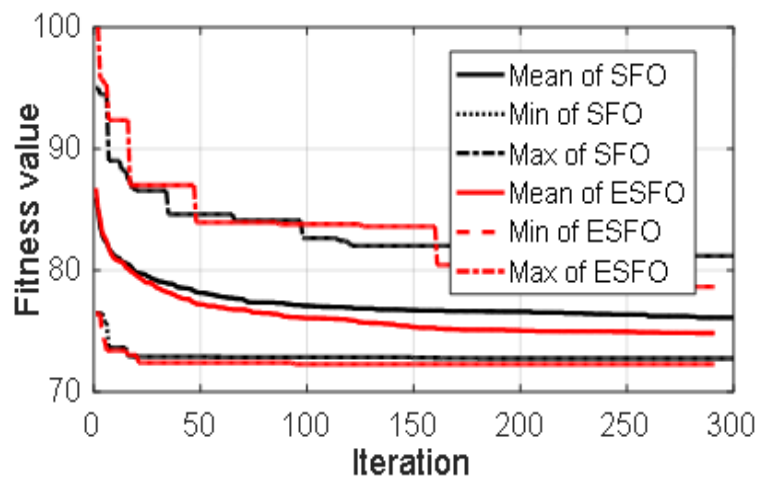

(b)

Figure 4. Comparison of performance of ESFO and SFO, (a) Optimal fitness value in 50 runs and (b) Convergence curves in 50 runs

\section{CONCLUSION}

In this paper, ESFO has been successfully proposed for the DG optimization problem to minimize active power loss of the distribution system. In which, ESFO has been added the mutation technique for updating the best sunflower plant. The performance of the proposed ESFO has been evaluated on the 33 nodes test system. The obtained results compared with the original SFO have sent a message that ESFO outperforms to SFO in terms of the minimum power loss as well as indexes related to effectiveness of an optimization algorithm such as maximum, minimum and mean values as well as STD of the fitness function. The compared results with other previous methods have also leaded to the conclusion that ESFO is in one of the effective techniques to the DG optimization problem for power loss reduction. Thus, ESFO can be a potential method for solving the DG optimization problem for practical systems or gratifying other goals. 


\section{REFERENCES}

[1] K. Alanne and A. Saari, "Distributed energy generation and sustainable development," Renewable and Sustainable Energy Reviews, vol. 10, no. 6, pp. 539-558, 2006

[2] S. M. M. Khormandichali and M. A. Kamarposhti, "Optimal placement of wind generation units in order to increase revenues and reduce the imposed costs in the distribution system considering uncertainty," International Journal of Electrical and Computer Engineering (IJECE), vol. 9, no. 6, pp. 4524-4539, 2019

[3] A. Musa and T. J. Tengku Hashim, "Optimal sizing and location of multiple distributed generation for power loss minimization using genetic algorithm," Indonesian Journal of Electrical Engineering and Computer Science (IJEECS), vol. 16, no. 2, pp. 956-963, 2019.

[4] A. Hoballah, Y. Ahmed, and K. A. Shoush, "Optimal utilization of automated distributed generation in smart grid using genetic algorithm," Indonesian Journal of Electrical Engineering and Computer Science (IJEECS), vol. 16, no. 1, pp. 82-91, 2019.

[5] M. E. Amran et al., "Optimal distributed generation in green building assessment towards line loss reduction for Malaysian public hospital," Bulletin of Electrical Engineering and Informatics (BEEI), vol. 8, no. 4, pp. 1180-1188, 2019.

[6] N. Ghadimi, "Using HBMO Algorithm to Optimal Sizing \& Sitting of Distributed Generation in Power System," Bulletin of Electrical Engineering and Informatics (BEEI), vol. 3, no. 1, pp. 1-8, 2014.

[7] M. H. Moradi and M. Abedini, "A combination of genetic algorithm and particle swarm optimization for optimal DG location and sizing in distribution systems," International Journal of Electrical Power \& Energy Systems, vol. 34, no. 1, pp. 66-74, 2012.

[8] B. Mukhopadhyay and D. Das, "Multi-objective dynamic and static reconfiguration with optimized allocation of PV-DG and battery energy storage system," Renewable and Sustainable Energy Reviews, vol. 124, 2020.

[9] M. N. Morshidi, et al., "Whale optimization algorithm based technique for distributed generation installation in distribution system," Bulletin of Electrical Engineering and Informatics (BEEI), vol. 7, no. 3, pp. 442-449, 2018.

[10] J. P. Sridhar and R. Prakash, "Multi-objective whale optimization based minimization of loss, maximization of voltage stability considering cost of DG for optimal sizing and placement of DG," International Journal of Electrical and Computer Engineering (IJECE), vol. 9, no. 2, pp. 835-839, 2019.

[11] S. M. Ali, P. S. Babu, and B. Gurusekhar, "Reconfiguration with Simultaneous DG installation to Improve the Voltage Profile in Distribution Network using Harmony Search Algorithm," Bulletin of Electrical Engineering and Informatics (BEEI), vol. 4, no. 4, pp. 257-273, 2015.

[12] R. S. Rao, et al., "Power Loss Minimization in Distribution System Using Network Recon fi guration in the Presence of Distributed Generation," IEEE Transaction on Power System, vol. 28, no. 1, pp. 317-325, 2013.

[13] M. Abdelbadea, T. A. Boghdady, and D. K. Ibrahim, "Enhancing active radial distribution networks by optimal sizing and placement of DGs using modified crow search algorithm," Indonesian Journal of Electrical Engineering and Computer Science (IJEECS), vol. 16, no. 3, pp. 1179-1188, 2019.

[14] T. T. Nguyen, A. V. Truong, and T. A. Phung, "A novel method based on adaptive cuckoo search for optimal network reconfiguration and distributed generation allocation in distribution network," International Journal of Electrical Power \& Energy Systems, vol. 78, pp. 801-815, 2016.

[15] A. M. Imran, M. Kowsalya, and D. P. Kothari, "A novel integration technique for optimal network reconfiguration and distributed generation placement in power distribution networks," International Journal of Electrical Power and Energy Systems, vol. 63, pp. 461-472, 2014.

[16] T. N. Ton, et al., "Optimal location and size of distributed generators in an elecric distribution system based on a novel metaheuristic algorithm," Engineering, Technology \& Applied Science Research, vol. 10, no. 1, pp. 5325-5329, 2020.

[17] A. Bayat, A. Bagheri, and R. Noroozian, "Optimal siting and sizing of distributed generation accompanied by reconfiguration of distribution networks for maximum loss reduction by using a new UVDA-based heuristic method," International Journal of Electrical Power and Energy Systems, vol. 77, pp. 360-371, 2016.

[18] M. R. Nayak, "Optimal Feeder Reconfiguration of Distribution System with Distributed Generation Units using HC-ACO," International Journal on Electrical Engineering and Informatics, vol. 6, no. 1, pp. 107-128, 2014.

[19] A. V. Truong, et al., "Two states for optimal position and capacity of distributed generators considering network reconfiguration for power loss minimization based on runner root algorithm," Energies, vol. 12, no. 1, 2019.

[20] R. Rajaram, K. Sathish Kumar, and N. Rajasekar, "Power system reconfiguration in a radial distribution network for reducing losses and to improve voltage profile using modified plant growth simulation algorithm with Distributed Generation (DG)," Energy Reports, vol. 1, pp. 116-122, 2015.

[21] N. Khalesi, N. Rezaei, and M. R. Haghifam, "DG allocation with application of dynamic programming for loss reduction and reliability improvement," International Journal of Electrical Power and Energy Systems, vol. 33, no. 2, pp. 288-295, 2011.

[22] A. Keane and M. O'Malley, "Optimal allocation of embedded generation on distribution networks," IEEE Transactions on Power Systems, vol. 20, no. 3, pp. 1640-1646, 2005.

[23] Y. M. Atwa, et al., "Optimal Renewable Resources Mix for Distribution System Energy Loss Minimization,” IEEE Transactions on Power Systems, vol. 25, no. 1, pp. 360-370, 2010.

[24] G. F. Gomes, S. S. da Cunha, and A. C. Ancelotti, "A sunflower optimization (SFO) algorithm applied to damage identification on laminated composite plates," Engineering with Computers, vol. 35, no. 2, pp. 619-626, 2019.

[25] M. E. Baran and F. F. Wu, "Network reconfiguration in distribution systems for loss reduction and load balancing," IEEE Transactions on Power Delivery, vol. 4, no. 2, pp. 1401-1407, 1989. 